Ann. Zootech., I977, 26 (3), 329-344.

\title{
Influence de 1'étalon sur la fertilité dans l'élevage des chevaux de sang
}

\author{
B. LANGLOIS \\ avec la collaboration de \\ I. Cattraux, D. Poirei et D. Tastu \\ Station de génétique quantitative et appliquée, \\ Centre National de Recherches Zootechniques, I.N.R.A., \\ 78350 Jouy-en-Josas (France)
}

\begin{abstract}
Résumé
Trois variables caractéristiques de la fertilité annuelle des étalons ont été analysées sur 352 mâles de race Anglo-Arabe, Pur Sang Anglais, Trotteur Français et Selle Français, répartis dans les Haras Nationaux d'Angers, Annecy, Blois, Cluny, La Roche-sur-Yon, Le Pin et Saint-Lô. Ils avaient au minimum 2 ans de service correspondant à au moins $3^{\circ}$ juments saillies.

Les analyses ont été effectuées sur deux échantillons : l'un faisant intervenir les 352 étalons totalisant I 505 années de monte; l'autre composé de 248 étalons sélectionnés sur un minimum de 25 juments saillies dans l'année, totalisant alors 699 années de monte.

L'analyse " hiérarchique " de la variance (voir le modèle dans le texte) a permis de révéler successivement :

- un effet de circonscription sur chaque variable pour l'échantillon complet et sur deux d'entre elles après troncature. Cet effet expliquant de l'ordre de 3 à $4 \mathrm{p}$. Ioo des variances pour l'ensemble des données s'est trouvé augmenté à 6 à $7 \mathrm{p}$. roo par réduction de la variance résiduelle pour l'échantillon tronqué,

- un effet race intra-circonscription noté de l'ordre de 2 à 3 p. Ioo de la variance sur deux variables dans la première étude ne s'est plus manifesté que sur l'une d'elles dans la seconde,

- un effet individuel de l'étalon qui s'est montré en revanche hautement significatif dans tous les cas. Expliquant de l'ordre de is p. ioo de la variance pour l'échantillon complet il en explique de l'ordre de 25 p. roo dans le second. Les répétabilités correspondantes de la fertilité annuelle de l'étalon comprises entre $0, I_{5}$ et 0,20 dans le premier cas peuvent atteindre la valeur 0,40 dans le second, lorsque les étalons saillissent un minimum de 25 juments par an.

Une analyse de variance "croisée " (voir le modèle dans le texte) a ensuite été conduite sur les deux mêmes échantillons. Elle a permis de confirmer les effets de la circonscription et de la race et $n$ 'a pas montré dans les conditions françaises d'in fluence significative du nombre de juments saillies et de l'âge des étalons.
\end{abstract}

\section{Introduction}

L'amélioration de la productivité de l'élevage est pour des raisons économiques l'un des principaux objectifs de la zootechnie. Dans cette optique, la maîtrise des phénomènes de la reproduction constitue un problème essentiel sur lequel de nombreuses recherches sont effectuées. En liaison avec de nouvelles techniques d'éle- 
vage, elles ont permis d'obtenir des taux de fertilité de l'ordre de 70 à 90 p. roo dans la plupart des espèces domestiques. Les chevaux, en revanche, n'ont pas connu la même évolution, ce taux très variable oscillant selon les populations entre 40 et 85 p. Ioo. De gros progrès étant envisageables, un programme d'études a été mis en place en France par les Haras Nationaux en liaison avec l'Institut National de la Recherche Agronomique. Les points déjà abordés sont le contrôle du cycle sexuel de la jument et le groupage des chaleurs (PALMER et Jousset, I975), les diagnostics précoces de gestation (PALMER, Thimonier et Lemon, I974), les techniques de détection des chaleurs et la monte en liberté (ARchambeaud et al., I973). Le problème de la fertilité des étalons n'a pas encore été approfondi. Il nous a donc paru utile, par une analyse statistique des résultats de la monte, d'essayer de discerner la part qui revient au mâle dans les variations de la fertilité. Ce paramètre est en effet une caractéristique d'élevage qui subit l'influence de nombreux facteurs de variations qu'on s'accorde généralement à répartir en facteurs " intrinsèques " ou " endogènes " c'est-à-dire propres aux animaux et en facteurs " extrinsèques » ou " exogènes " c'est-à-dire liés principalement aux conditions de la fécondation et aux techniques d'élevage. Dans notre cas, c'est surtout l'importance des facteurs " intrinsèques " propres aux étalons, compte tenu des effets de la région, de la race, de l'âge et du nombre de juments saillies, que nous proposons de déterminer, afin éventuellement d'en justifier une analyse physiologique plus approfondie. Notre démarche se veut donc exclusivement descriptive et ses résultats ne devront pas être extrapolés à d'autres conditions d'utilisation des étalons que celles qui sont actuellement réalisées en France (" monte en main " d'un nombre limité de juments).

\section{Matériel et méthodes}

\section{I. - Matériel animal}

Nous disposions pour notre enquête des archives des Haras Nationaux. Les documents étudiés ont été les "registres des étalons " établis à partir des "états des chefs de station ". Ces états parviennent aux Haras un an après la saison de monte considérée puisqu'ils contiennent les résultats des saillies. Ce sont les seuls documents qui mentionnent les femelles vides, avortées, mortes, vendues ou sans renseignements. Les "fiches de saillies" accompagnées des " déclarations de naissance " ont été également consultées et comparées aux résultats du registre à titre de vérification et d'amélioration de la précision. Les informations recueillies par étalon et par année ont été : la circonscription de monte, la race, l'âge, le nombre de juments saillies, n'ayant pas retenu, ayant avorté, dont on était sans renseignements et le nombre de mâles et de femelles obtenus. A l'origine, l'enquête devait tenir compte de la "station de monte " et du " palefrenier chef de station " mais les effets de ces facteurs de variation étant le plus souvent confondus avec celui de l'étalon, ils n'ont pu être pris en considération. Les états concernant les étalons privés, comportant trop de lacunes sur le résultat des saillies, ont été également laissés de côté. Seuls les étalons nationaux qui ont l'avantage de bénéficier de conditions de milieu relativement homogènes ont donc été pris en considération. Sept Haras ont été visités (Angers, Annecy, Blois, Cluny, La Rochesur-Yon, Le Pin et Saint-Lô) et 4 races retenues : l'Anglo-Arabe (AA), le Pur Sang Anglais (PS), le Trotteur Français (TF) et le Selle Français (SF). La race angloarabe, concentrée surtout dans le sud-ouest de la France, n'a donc pas ici été 
abordée de façon tout à fait représentative. Dans l'ensemble, les 357 étalons dont nous avons relevé les résultats avaient au minimum 2 ans de service correspondant à au moins 30 juments saillies. Au total I 505 résultats annuels ont ainsi été recueillis, représentant la monte de 24 étalons AA, I05 PS, 89 TF et I39 SF.

\section{2. - Méthodes}

I. Les variables.

Des difficultés apparaissent lorsque l'on aborde la fertilité des étalons. Elles sont dues aux nombreux facteurs intervenant, au nombre élevé de juments sans renseignements, aux informations parfois imprécises sur le résultat des saillies (les juments vides ou avortées ne sont pas toujours bien déterminées). Malgré la complexité du choix des variables, nous en avons retenu 3 :

- FER $\mathrm{I}=\frac{\text { nombre de produits }}{\text { nombre de produits }+ \text { nombre de juments vides }} \times$ Ioo

- FER $2=\frac{\text { nombre de produits }}{\mathrm{nb} \text { de produits }+\mathrm{nb} \text { de juments vides }+ \text { nb de juments avortées }} \times$ roo

- FER $3=\frac{\text { nombre de produits }}{\text { nombre de juments saillies }} \times$ IoO

Chacune d'elles permet d'estimer les caractéristiques de la fertilité du mâle, mais aucune ne représente sa fertilité réelle et il est difficile de déterminer celle qui est la plus exacte. Par ailleurs, d'autres variables susceptibles d'apporter plus d'informations à l'étude étaient à notre disposition. Elles ont donc été prises en considération. Il s'agit :

- du taux de juments sans renseignements

$$
\mathrm{SREN}=\frac{\text { nombre de juments sans renseignements }}{\text { nombre de juments saillies }} \times \text { roo }
$$

- du nombre de juments saillies

NBTS $=$ nombre de juments saillies

- du taux de masculinité

$$
\text { TAMA }=\frac{\text { nombre de mâles }}{\text { nombre de mâles }+ \text { nombre de femelles }} \times \text { Ioo }
$$

Au total, 6 variables vont donc intervenir dans les analyses.

\section{Les analyses statistiques}

- Une analyse hiérarchique selon le modèle suivant a été appliquée aux 6 variables pour estimer les effets de la circonscription, de la race et de l'étalon :

$$
\mathrm{X}_{i j k l}=\mu+\mathrm{C}_{i}+\mathrm{R}_{i j}+\mathrm{E}_{i j k}+r_{i j k l}
$$

dans lequel

$\mathrm{X}_{i j k l}$ est la variable correspondante à une année de monte.

$\mu \quad$ est la moyenne générale.

$\mathrm{C}_{i} \quad$ représente l'effet de la ieme circonscription. 
B. LANGLOIS

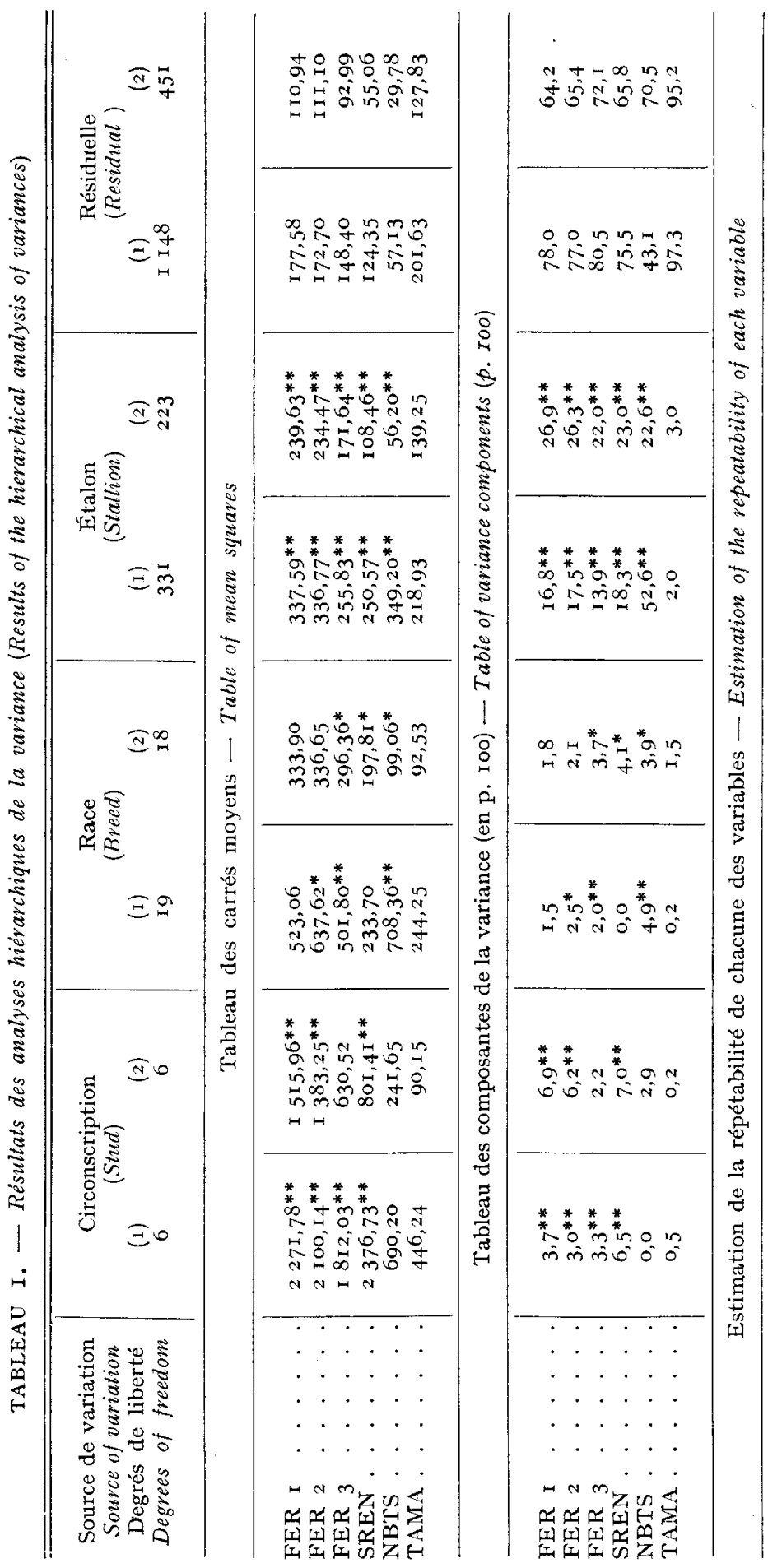

유 원

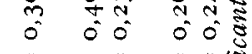

\| $\|$ \|ा $\|\mid\|$.

(is

N

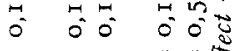

|| || || || ||

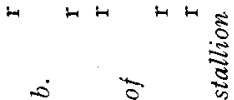

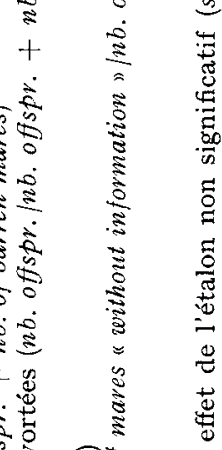

कृ

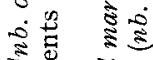

เ.

क⿺ है

द 5 कि

官+

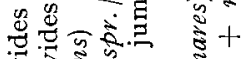

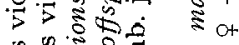

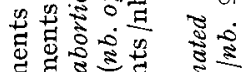

过苛苞

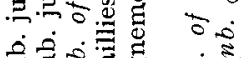

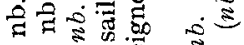

$++i \infty$ 业

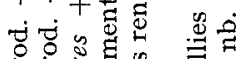

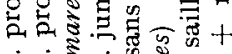

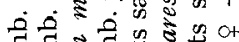

द्व

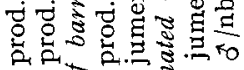

ف을

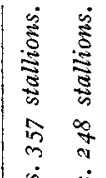

ริ

दे इ

के हे

$\because \quad 0$

ปี

胥

$\stackrel{2}{2}$

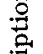

逑

है

品

¿

(1)

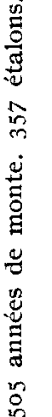

i 8

....

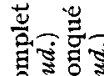

... .

ing

. .

. . . . .

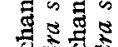

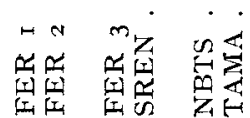

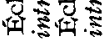

제

$\begin{array}{ll}5 & 5 \\ 0 & 0\end{array}$ 
$\mathbf{R}_{i j} \quad$ représente l'effet de la race $j$ à l'intérieur de la circonscription $i$.

$\mathrm{E}_{i j k}$ représente l'effet du $k^{e m e}$ étalon dans la circonscription $i$ et la race $j$.

$\gamma_{i j k l}$ représente l'effet résiduel de l'année $l$ sur l'étalon $k$ dans la circonscription $i$ et la race $j$.

Notons que la classification hiérarchique n'est pas strictement respectée pour les races. Nous considérons ici des combinaisons race-circonscription qui, de plus, sont parfois faiblement représentées.

Par ailleurs, ce modèle autorise l'estimation des composantes de la variance et donc de la répétabilité de la performance annuelle d'un étalon par le rapport de la composante étalon sur la somme des composantes étalon et résiduelle.

- Dans un second temps, pour vérification des effets de la race et de la circonscription et pour détermination de l'effet de l'âge et du nombre de juments saillies, les variables FER I, FER 2, FER 3, SREN et TAMA ont été classées en fonction de l'âge (I7 niveaux de 4 ans à 20 ans et plus), de la circonscription (7 niveaux), du nombre de juments saillies ( 7 niveaux par classe de ro de o à 70 puis 5 niveaux par classe de Io de 20 à 70 ), de la race de l'étalon (4 niveaux) pour être soumises à une analyse de la variance par la méthode des moindres carrés selon le modèle :

$$
\mathrm{X}_{i j k l}=\mu+\mathrm{A}_{i}+\mathrm{C}_{j}+\mathrm{S}_{k}+\mathrm{R}_{l}+r_{i j k l}
$$

dans lequel

$\mathrm{X}_{i j k l}$ est la variable correspondante à une année de monte.

$\mu \quad$ est la moyenne générale.

$\mathrm{A}_{i} \quad$ représente l'effet de l'âge $i$.

$\mathrm{C}_{j} \quad$ représente l'effet de la circonscription $j$.

$\mathrm{S}_{k} \quad$ représente l'effet de la classe $k$ du nombre de juments saillies.

$\mathrm{R}_{l} \quad$ représente l'effet de la race $l$.

$r_{i j k l}$ représente la résiduelle qui est une variable aléatoire de moyenne nulle.

- Ces analyses ont été appliquées à deux échantillons, l'un étant représenté par la totalité des étalons du fichier, l'autre étant constitué par les mâles ayant un minimum de 25 juments saillies par saison de monte. Ceci de façon à éliminer une part de variation aléatoire sur l'ensemble des pourcentages étudiés.

\section{Résultats}

\section{I. - Effets relatifs de la région, de la race et de l'étalon}

Le tableau I donne l'ensemble des résultats des analyses hiérarchiques correspondantes.

Sur l'échantillon complet interviennent I 505 années de monte, 357 étalons, 26 combinaisons race-circonscription et 7 circonscriptions. Sur le second échantillon sélectionné sur un nombre annuel de juments saillies supérieur à 25 , les effectifs se réduisent respectivement à $699,248,25$ et 7 .

\section{"L'effet circonscription"}

Il s'est manifesté pour les deux échantillons sur les variables FER I et FER 2 expliquant respectivement 3,4 puis 6,9 p. Ioo et 3 puis $6,2 \mathrm{p}$. Ioo de leur variance. Il en est de même pour la variable $S R E N$ où il représente 6,5 puis $7 \mathrm{p}$. Ioo de la variance. Nous remarquons donc dans la deuxième analyse une augmentation de l'ordre de 3 p. roo pour les deux variables de la fertilité et un niveau d'action à peu près équivalent pour le taux de "sans renseignements " (SREN). En revanche, 
l'effet circonscription sur la variable FER 3 étant passé de 3,3 à 2,2 p. Ioo de sa variance, il n'apparaît significatif que dans la première analyse. Aucune action de ce facteur n'a par ailleurs été mise en évidence sur le nombre de juments saillies par étalon (NBTS) ni sur le taux de masculinité (TAMA).

\section{"L'effet race"}

L'effet de la race de l'étalon s'est manifesté dans la première étude sur les variables FER 2 et FER 3 ( 2 à 2,5 p. Ioo de la variance). L'effet sur FER 2 diminuant sensiblement (- -4 p. IOO) apparaît non significatif dans la deuxième étude. L'absence d'effet de ce facteur sur FER I semblerait indiquer que son action se situerait plutôt au niveau du taux d'avortement ou de mortalité embryonnaire. Sur la variable SREN, son influence un peu plus marquée dans la seconde analyse qu'elle n'était dans la première ( +4 , I p. Ioo), fait qu'elle devient alors significative. Le nombre de juments saillies (NBTS) s'est révélé dans les deux études être influencé par la race qui explique 4 à 5 p. Ioo de sa variance. D'après l'échantillon complet, les moyennes par race se situent ainsi : PS 33,9 juments saillies/an, AA 30,5, TF 30,2 et SF 29,9 .

\section{"L'effet étalon »}

Il s'est montré dans les deux cas hautement significatif sur toutes les variables sauf le taux de masculinité (TAMA). Dans la seconde analyse, la sélection des années de monte sur un nombre de juments supérieur à 25 , a réduit dans une proportion importante la variance d'origine purement aléatoire de la plupart des variables. Elle a donc proportionnellement augmenté la part due à l'étalon. C'est ainsi que les facteurs liés à l'étalon expliquent respectivement pour les variables FER I, FER 2, FER 3 et SREN, Io, 9, 8 et 5 p. Ioo de variance en plus dans la seconde analyse que dans la première. Cela porte leur influence à respectivement $27,26,22$ et 23 p. Ioo de la variance de ces variables. En revanche, dans la seconde, analyse, l'influence de l'étalon sur le nombre de juments saillies baisse de l'ordre de 30 p. roo, ce qui est la conséquence normale de l'homogénéisation de cette variable réalisée dans cet échantillon.

Les valeurs de répétabilité obtenues pour les trois taux de fertilité FER I, FER 2 et FER 3 sont respectivement de o, I 8, o, I9 et $0, I_{5}$ dans l'analyse de l'échantillon complet. En sélectionnant les étalons qui saillissent régulièrement plus de 25 juments, elles atteignent 0,30 pour FER $1,0,40$ pour FER 2 et 0,23 pour FER 3. Par ailleurs le nombre annuel de juments saillies par étalon (NBTS) se révèle très stable ( $r=0,55$ sur l'échantillon complet).

Ainsi, dans ces deux analyses, les variables liées à la fertilité soit FER $\mathrm{r}$, FER 2, FER 3 et même SREN sont influencées d'abord par l'étalon dont l'effet est nettement prépondérant, ensuite par la circonscription dont l'effet est le plus souvent significatif et ensuite assez faiblement par la race.

La variable nombre de juments saillies (NBTS) est très dépendante de l'étalon et influencée dans une proportion beaucoup plus faible par la race.

Le taux de masculinité (TAMA) ne subit aucune influence.

\section{2. - Les analyses "croisées " de la variance}

Le tableau 2 donne l'ensemble des résultats sur les deux échantillons utilisés.

- le test global des interactions qui permet de déterminer si le modèle d'analyse choisi en supposant l'additivité des effets des facteurs de variation peut être 


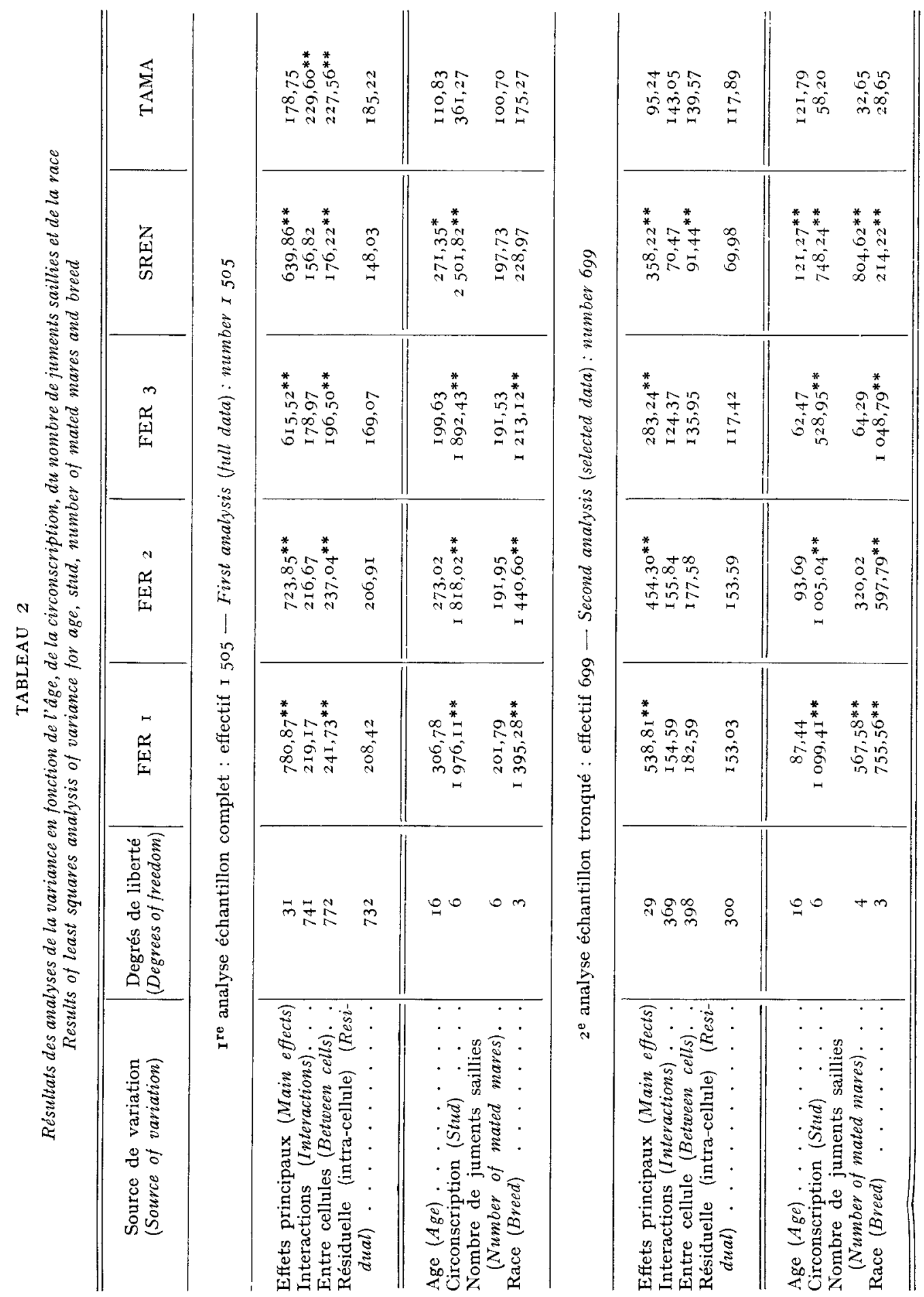


retenu, s'est avéré très largement concluant sauf pour le taux de masculinité (TAMA) dans l'échantillon complet. Pour cette variable, l'étude des facteurs de variation deux à deux a permis de localiser la source de cette interaction entre circonscription et nombre de juments saillies d'une part et entre nombre de juments saillies et race d'autre part. Ceci nous a amené à penser que vraisemblablement le sexe des produits n'était pas toujours dans certains haras relevé de façon précise,

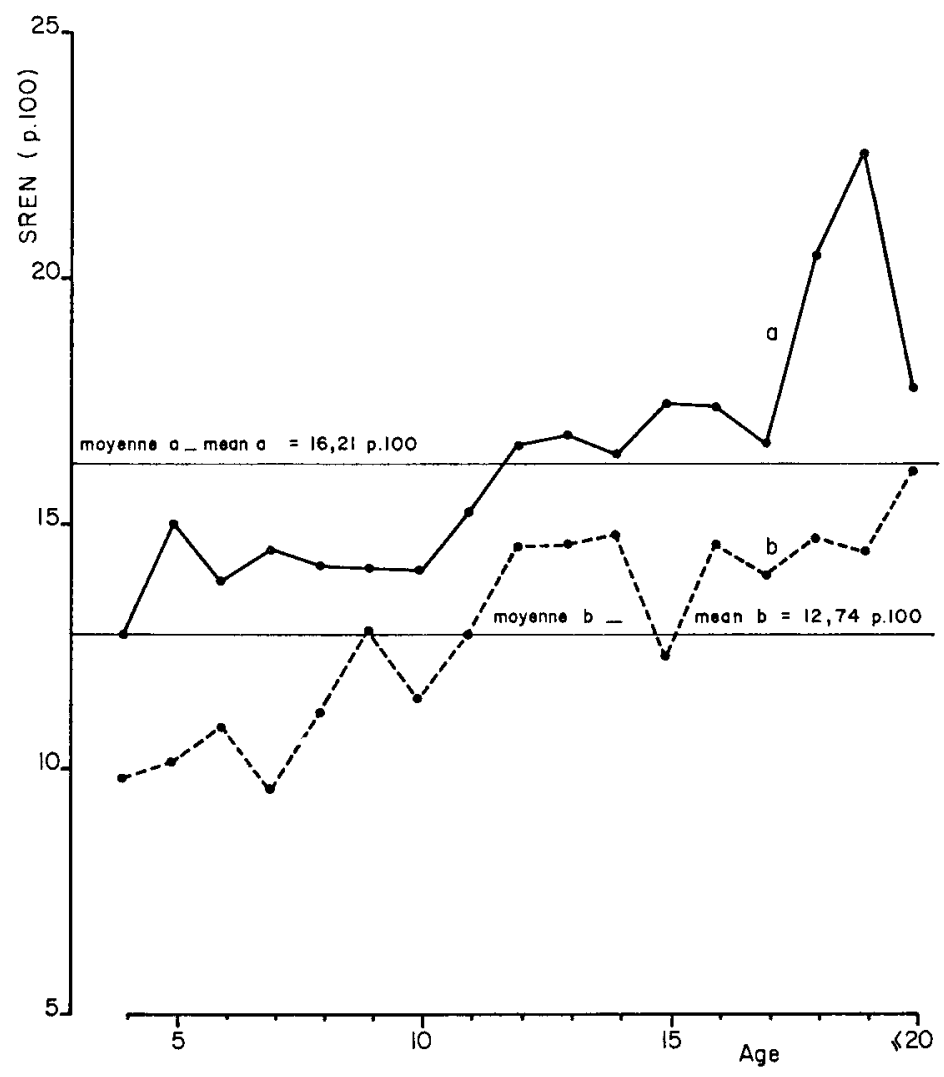

Frg. I. - Effet de l'âge sur le taux de "sans venseignements" (SREN). Effect of age on the rate of mares "without information" (SREN).

$\mathrm{a}$ : données complètes (full data)

b : données tronquées (selected data)

surtout lorsque le nombre de juments saillies était important, les vérifications étant alors plus laborieuses, et lorsque les races considérées étaient peu contrôlées comme dans le cas de l'élevage pour la selle.

- la composante de la variance "entre-cellules", soit entre les groupes d'observations concernant le même âge, la même circonscription, le même niveau du nombre de juments saillies et la même race, s'est montrée significative pour toutes les variables lors de la première analyse. Dans la seconde, du fait de l'homogénéité des données résultant du seuil de 25 juments imposé à chaque année de monte, elle ne l'est plus que pour le taux de "sans renseignements" (SREN).

Les effets principaux, soit de l'ensemble des facteurs de variation testés, se 
sont manifestés sur toutes les variables dans les deux analyses sauf pour le taux de masculinité qui n'apparaît en résumé influencé par aucun des facteurs étudiés. Pour les autres variables, la décomposition de ces effets, facteur par facteur, est la suivante :

\section{"Effet de l'âge"}

Il ne s'est manifesté sur aucune des trois variables de fertilité. Il se révèle cependant significatif sur le taux de "sans renseignements" (SREN) dans chacune des deux analyses (fig. I).

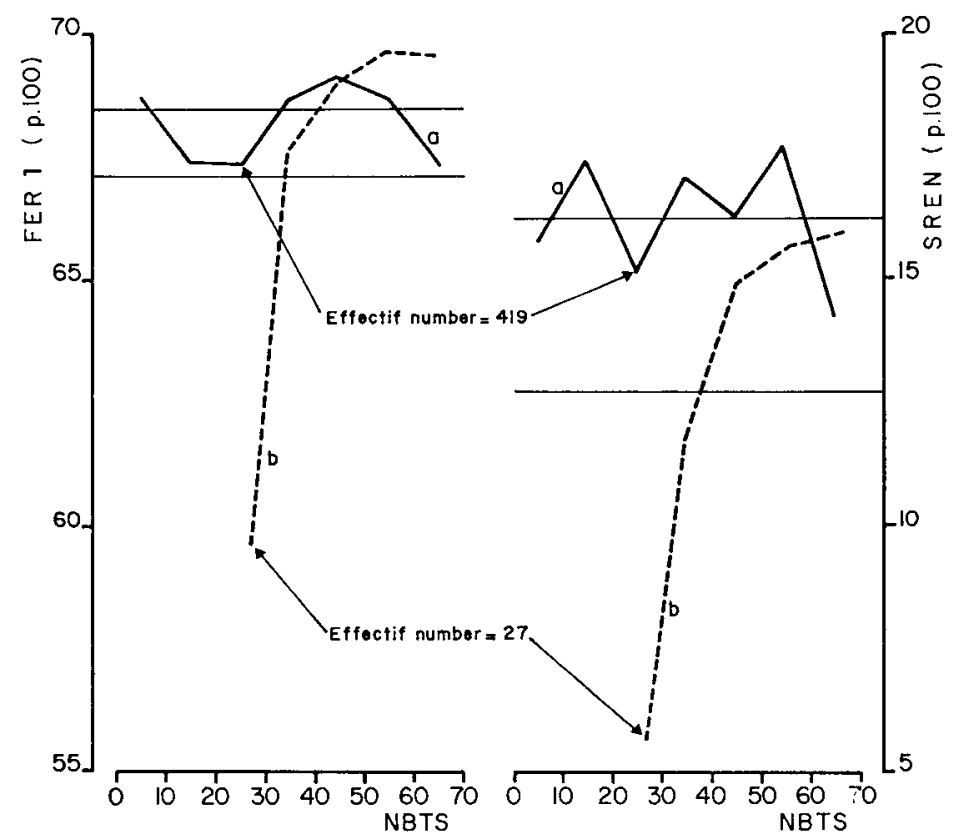

FIG. 2. - Effet du nombre de juments saillies (NBTS) sur la fertilité (FER I) et sur le taux de "sans renseignements " (SREN).

Effect of the number of mated mares (NBTS) on fertility (FER I) and on the rate of mares "without information" (SREN).

a : données complètes (effets non significatifs) full data (not significant),

$\mathrm{b}$ : données tronquées (effets significatifs) selected data (significant),

\section{"Effet de la circonscription"}

Les résultats obtenus par l'anayse hiérarchique se trouvent confirmés par ces deux nouvelles analyses.

\section{"Effet du nombre de juments saillies"}

Un effet du nombre de juments saillies n'est apparu que dans la seconde analyse sur les variables FER I et SREN. Il semble que dans ce cas (fig. 2) il soit dû aux faibles performances de la classe 25 à 30 juments saillies dont l'effectif, du fait de la troncature de l'échantillon, n'est que de 27 années de monte. Ce niveau 
faiblement représenté étant supprimé, l'effet ne serait très certainement pas apparu. Il n'y a donc pas lieu de penser dans les limites de cette étude que le nombre de juments saillies affecte la fertilité des étalons.
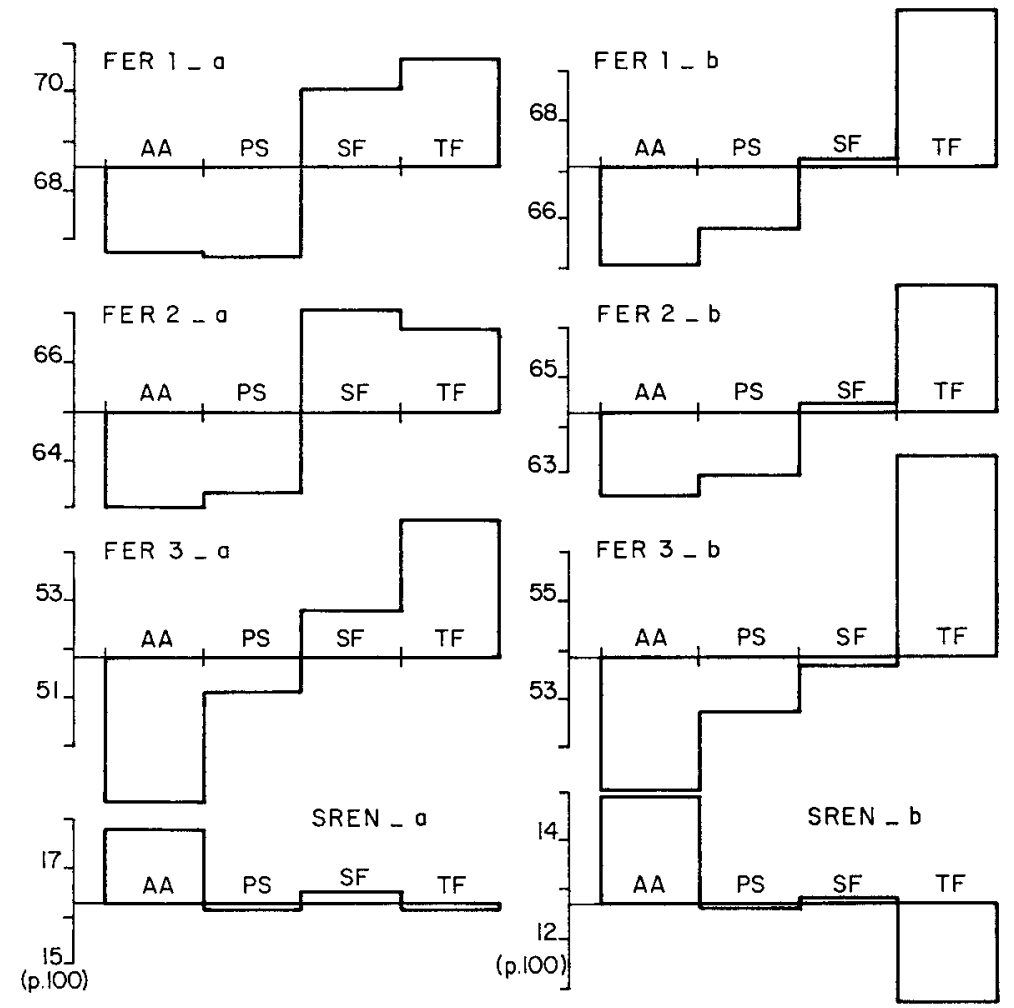

FIG. 3. - Effet de la vace de l'étalon sur les variables de fertilité (FER I, FER 2, FER 3) et sur le taux de "sans renseignements " (SREN).

Effect of the breed of the stallion on its fertility level (FER $I, F E R$ 2, FER 3) and rate of mares "without information" (SREN).

a : données complètes (full data)

AA : Anglo-Arabe

AA : Anglo-Arab

SF : Selle Français

$\mathrm{SF}$ : French saddlebred b : données tronquées (selected data)

PS : Pur Sang Anglais

PS : Thoroughbred

TF : Trotteur Français

TF : French Trotter

\section{"Effet de la race de l'étalon"}

Comme ceux de la circonscription, les effets de la race se sont confirmés dans ces deux analyses. Les étalons Trotteur Français et Selle Français apparaissent légèrement plus fertiles que les étalons Pur Sang Anglais et Anglo-Arabe (fig. 3).

\section{Discussion}

Nous aborderons successivement l'examen critique des variables estimateurs de la fertilité des étalons, la discussion de la fiabilité des données de base utilisées et la portée pratique des résultats obtenus compte tenu de la confusion de certains effets de milieu avec celui de l'étalon. 


\section{I. - Choix des variables}

Le nombre important de juments sans renseignements fait qu'on ne dispose pas d'estimateur sans erreur systématique de la fertilité des étalons. En effet, bien qu'il apparaisse sur l'ensemble des effets observés que le taux de juments sans renseignements évolue à l'inverse des variables de fertilité, il n'est pas possible d'apprécier la proportion de femelles vides qu'il représente même s'il semble que ce soit la majorité. De ce fait, les taux moyens de FER I, FER 2 et FER 3 qui sont respectivement de $0,68,0,65$ et 0,52 ne reflètent pas rigoureusement la fertilité moyenne de l'élevage qu'il semble raisonnable de situer vers 0,55 . Notons encore que si nous entendons par fertilité d'un étalon sa probabilité de fertiliser une jument, les données dont nous pouvons disposer, soit les comptes rendus annuels, excluent l'analyse fine du phénomène. Le nombre de sauts pour chaque jument ainsi que leur repérage par rapport aux chaleurs seraient dans ce cas des renseignements indispensables. Remarquons à ce propos que Bellitri et Pagano (I967) exploitant des informations de ce type, ont pu mettre en évidence des effets hautement significatifs de l'étalon sur le nombre de saillies par conception et sur l'intervalle premier service-fécondation. Néanmoins, malgré l'imprécision des données disponibles pour notre étude, il s'est avéré possible de caractériser chaque étalon par les variables FER I, FER 2 et FER 3 dont les répétabilités voisines de $0, \mathrm{I} 7$ sur le premier échantillon peuvent atteindre la valeur de 0,40 pour FER 2 sur le second échantillon, lorsqu'une partie des fluctuations aléatoires dues aux faibles effectifs se trouve éliminée. Remarquons encore que la supériorité de la répétabilité de FER 2 la désigne pour caractériser au mieux les variations de fertilité entre étalons. En effet, FER 3 qui paraît a priori moins biaisée, subit du point de vue de la variation l'effet des juments "sans renseignements ». Or, nous l'avons vu, les raisons de ces "sans renseignements " sont diverses, échecs de fertilité certes mais également mort, vente, changement de circonscription, exportation... Cette multiplicité de facteurs de variation retentit défavorablement sur la répétabilité de ce critère et fait que malgré le plus grand biais systématique dont est l'objet la variable FER 2, elle est préférable aux deux autres. Cependant si l'on se donne la valeur 0,70 comme seuil pour le coefficient de détermination $k$ du potentiel de l'étalon (*), il faut en moyenne ro ans, dans les conditions actuelles de reproduction, pour l'atteindre.

Cette durée moyenne pour la population varie évidemment beaucoup d'un étalon à un autre selon le nombre de juments saillies. Illustrant ce fait, le même calcul que précédemment, en sélectionnant les étalons qui saillissent régulièrement plus de 25 juments par an $(r=0,40$ pour FER 2$)$, montre que la durée de Io ans est ramenée à moins de 4 ans. En raison de la forte imprécision des variables disponibles, si l'on désire malgré tout (U.N.I.C. I97I à 1975) fournir des statistiques de fertilité par étalon, nous ne pouvons recommander que les 4 points suivants:

- utiliser l'estimateur FER 2.

- prendre en compte la totalité de la carrière de l'étalon et non les seuls résultats annuels.

$$
k=\frac{n r}{\mathrm{I}+(n-\mathrm{T}) r}
$$

- $n$ est le nombre d'années de monte connues pour l'étalon.

-. $r$ est la répétabilité $(r=0,19$ pour FER 2). 
- fournir un intervalle de confiance fondé sur la distribution d'échantillonnage d'un pourcentage.

- exprimer les résultats en écarts à la moyenne générale.

\section{2. - Fiabilité des données}

Deux aspects sont à considérer ici : l'exactitude des renseignements recueillis et la représentativité de l'échantillon de travail. Les documents sur lesquels a été constitué notre fichier sont l'aboutissement d'opérations multiples où les possibilités d'erreurs ne sont pas à exclure. Ainsi par exemple des fautes sur le sexe sont à l'origine d'effets parasites dans notre étude. Dans l'ensemble, il semble cependant qu'elles se répartissent au hasard, ce qui ne nuit pas aux interprétations. La question de la représentativité de l'échantillon est plus difficile à évaluer. Un choix préférentiel des étalons ayant le plus servi a en effet été opéré et il est difficile de savoir s'il n'a pas conduit à choisir les mâles les plus fertiles réduisant ainsi la fraction de variance pouvant leur être imputée. Par race, pour l'AngloArabe notamment, l'échantillonnage peut aussi avoir introduit certains biais mais il est difficile pour des enquêtes conduites manuellement d'éviter ce genre d'écueil. A l'avenir, avec le fonctionnement informatique du système S.I.R.E. $\left({ }^{*}\right)$, nous pouvons entrevoir le renouvellement de ce genre d'études fondé sur des informations à la fois plus justes et plus complètes.

\section{3. - Incidence pratique des résultats}

Des effets dus à la circonscription et à la race, il faut retenir les différences sensibles entre Haras et la plus grande fertilité des mâles SF et TF. Toutefois leur importance, comprise entre 2 et 7 p. Ioo de la variance, se révèle modérée comparée à celle de l'étalon qui selon les variables se situe entre ${ }_{4} 4$ et $27 \mathrm{p}$. Ioo de cette même variance. Cette prépondérance est en accord avec les résultats de Schönmuth (I956) qui l'avait déjà soulignée. Il faut cependant insister sur la confusion dans notre analyse des effets strictement biologiques de l'étalon avec ceux de la "station de monte " et du "palefrenier chef de station ", l'une pouvant intervenir au niveau de la technicité moyenne des éleveurs de la région concernée, l'autre ayant bien entendu un rôle important au poste qu'il occupe. Si l'action de ces deux facteurs est importante (grandes différences de technicité des éleveurs et des chefs de station) il est possible que nous ayons surévalué la constance des résultats annuels de fertilité des étalons et de ce fait leur importance. Nous ne pensons pas cependant que cette éventuelle surévaluation soit très importante d'autant plus qu'un phénomène inverse pourrait être invoqué. En effet aucune influence de l'âge et du nombre de juments saillies, contrairement aux résultats de GeIst (I954), ne s'est manifestée ici. Ces résultats tout à fait conformes cependant à ceux de HarTwig (I954) doivent toutefois se nuancer du fait de l'augmentation régulière (fig. I) du taux de sans renseignements avec l'âge de l'étalon. En effet ces juments " sans renseignements " ( $16,2 \%$ des saillies) correspondent souvent à des échecs de reproduction. Cette augmentation de SREN avec l'âge pourrait donc être en partie interprétée comme une diminution de fertilité. Elle serait cependant d'une amplitude trop faible pour être mise significativement en évidence au niveau des varia-

(*) S.I.R.E. Système d'Identification Répertoriant les Equidés. 
bles de fertilité utilisées FER I, FER 2 et FER 3. Globalement cette absence des effets âge et nombre de juments saillies pourrait s'expliquer par le fait que dans les conditions françaises d'élevage, chaque étalon est exploité en fonction de ses possibilités. L'adaptation serait bonne puisque le nombre de juments saillies ne se répercute pas sur la fertilité, de même pour l'âge. Ces conditions, en revanche, tendraient à minimiser les différences entre étalons et nous conduiraient donc à sous évaluer leur importance potentielle.

Pour toutes ces raisons, un soin particulier apporté à la fertilité des mâles dans cette espèce ne nous paraît pas sans intérêt. Cela est d'autant plus souhaitable qu'une utilisation plus intensive des étalons est à rechercher sur le plàn génétique.

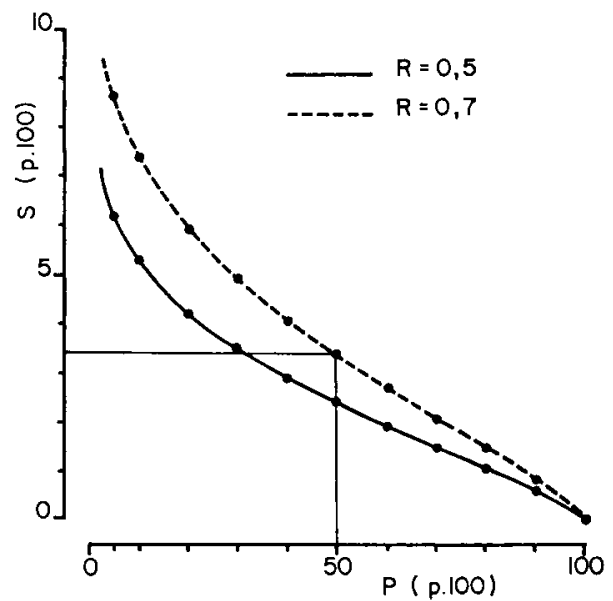

Fig. 4. - Augmentation de fertilité $S$, escomptée d'un taux $p$ de sélection des étalons, réalisé sue un critère d'estimation de précision $R$ :

- l'écart-type propre aux étalons du pourcentage de fertilité $\left(\sigma_{\mathbf{e}}\right)$ est supposé égal à 6 p. ıoo.

Increase of fertility $S$, anticipated from a selection rate $p$ of the stallions, made on a criterion of precision $R$ : 6 per cent.

- standard deviation of the fertility rate due to stallions $\left(\sigma_{v}\right)$ is supposed to be equal to N.B. - Au-dessus d'un taux de sélection de 0,50 le progrès à attendre sur la fertilité est inférieur à $3.5 \mathrm{p}$. IOo (for selection rates exceeding 0.50 , the increase of the fortility rate is less than 3.5 per cent).

Le développement de tests fondés sur l'analyse du sperme (Szumowski, I964) permettant d'estimer la fertilité des jeunes reproducteurs et autorisant ainsi leur sélection préalable, devrait conduire à une augmentation générale de fertilité dans l'élevage.

L'amplitude $S$ de cette augmentation attendue de l'application aux étalons d'un taux $p$ de sélection, peut être estimée par la formule suivante :

dans laquelle

$$
\mathrm{S}=\frac{z}{p} \sigma_{e} \mathrm{R}
$$

$z \quad$ est l'ordonnée de la courbe normale réduite au point de troncature correspondant à $p$.

$z / p=i$ est l'intensité de sélection réalisée. 
$\sigma_{e} \quad$ est la racine carrée (écart-type) de la variance de la fertilité propre à l'étalon. Dans notre cas pour la variable FER 2 sur les deux échantillons, $\sigma_{e}$ peut être approximativement évalué à 6 p. Ioo (racine carrée de la composante paternelle de la variance).

$\mathrm{R} \quad$ est la précision de l'estimateur de la fertilité utilisé, soit sa corrélation avec la véritable fertilité. $R$ est toujours inférieur à $I$ mais il est raisonnable d'espérer pouvoir bâtir à l'aide de plusieurs mesures, par des méthodes de régression multiplie, un estimateur dont la précision serait comprise entre $0,5^{\circ}$ et 0,70 .

I,a figure 4 permet de se rendre compte approximativement des progrès de fertilité envisageables par l'application systématique d'une telle méthode. Elle souligne l'importance de réaliser une sélection assez forte chez les futurs étalons (pas moins de $50 \mathrm{p}$. IoO) pour que les progrès soient sensibles au niveau général de l'élevage.

\section{Conclusion}

Dans cette étude l'effet de l'étalon, hautement significatif dans tous les cas, est apparu largement prépondérant sur tous les facteurs de variation étudiés (circonscription, race, nombre de juments saillies, âge). Expliquant de l'ordre de I5 p. Ioo de la variance pour l'échantillon complet, il en explique de l'ordre de $25 \mathrm{p}$. Ioo dans le second en raison de l'élimination d'une partic de variance d'origine aléatoire. Ce dernier chiffre laisse donc supposer malgré la confusion des effets "station de monte " et "palefrenier chef de station ", une importance non négligeable des effets liés aux mâles dans la fertilité générale de l'espèce dont la moyenne a pu être située à 0,55 .

La répétabilité de la fertilité annuelle des étalons sur l'échantillon complet estimée de l'ordre de o, I 8 a malheureusement montré que dans les conditions actuelles de reproduction, il faut en moyenne io ans pour connaître avec une précision suffisante le potentiel de chaque mâle. Il est donc pratiquement exclu de les sélectionner finement sur ce caractère. Toutefois si tous les étalons saillissaient plus de 25 juments par an, le délai précédent étant ramené à moins de quatre ans, cela ouvrirait certaines possibilités. Parallèlement, la recherche de critères plus analytiques fondés par exemple sur l'examen du sperme, devrait permettre une amélioration générale de fertilité à condition toutefois d'appliquer des taux rigoureux de sélection aux futurs étalons. Ceci devrait être possible en France, en raison du contrôle réglementaire de l'étalonnage, beaucoup plus sévère que ne l'est celui de la jumenterie.

Accepté pour publication en juin 1977.

\section{Summary}

\section{Stallion effect on fertility in " light-horse breeding "}

Three variables describing the annual fertility of stallions were analysed on 352 males of four breeds (Anglo-Arab, Thoroughbred, French Saddlebred and French Trotter) in seven national studs (Angers, Annecy, Blois, Cluny, La Roche-sur-Yon, Le Pin and Saint-Lô). When starting the study, the stallions had at least two years of activity corresponding to a minimum of 30 mated mares.

Analyses were made successively on two samples. The first one concerned $35^{2}$ stallions 
and totalized 1,505 yearly records. The second one concerned 248 stallions selected on the basis of at least 25 mares mated per year, leading to 699 yearly records.

The hierarchical analysis of variances (see the model in the text) revealed:

- a significant effect of the national stud on each variable of the first sample (full data) and on two of them after selection (selected data). This effect explained 3 to 4 per cent of the variances in the first sample and 6 to 7 per cent in the second one because of the reduction of residual variances,

- a breed effect (intra stud) significant for two variables in the first analysis $(2-3 \%$ of the variances) and only for one variable in the second analysis,

- a highly significant stallion effect for all variables explaining about i 5 per cent of the variance in the first sample and about 25 per cent in the second one. The corresponding repeatabilities of the stallion's animal fertility rates ranged between 0.15 and 0.20 in the first analysis and reached 0.40 in the second one when the males mated regularly a minimum of 25 mares per year.

A least squares analysis of variance (see model in the text) was then made on the two samples. It confirmed the " national stud " and " breed " effects. However, in French conditions of management the effects of the number of mated mares and of the age of the stallions were not significant.

\section{Références bibliographiques}

Archambeaud B., Jussiaux M., Palmer E., Signoret J. P., r973. Méthode de détection de l'oestrus chez la jument en liberté. Ann. Zootech., 22, 333-336.

Belliti E., Pagano M. G., I967. Indagine su alcuni parametri biologici del cavallo di Capitanata. Ann. Fac. Agric. Univ. Bavi, 21, I85-206.

Geist E., I954. Befruchtungsergebnisse und deren Schwankungen bei Hengsten des Niedersächsischen Landgestütes Celle. Thèse vétérinaive Justus. Liebig. Hochsch., Giessen, $4^{\circ}$ p. (Abstr. in Anim. breed. abstr., 24, $\mathrm{n}^{\circ}$ 966).

Hartwig W., I954. Untersuchungen über die Deck und Befruchtungsergebnisse der Beschäler des Landgestüts Kreuz unter besonderer Berücksichtigung der Befruchtungsfähigkeit der Hengste mit zunehmendem Alter. Kühn, Archiv., 68, 35-63. (Abstr. in Anim. breed. abstr., 23, no 37 ).

Palmer E., Jousset B., I975. Synchronization of oestrus and ovulation in the mare with a two PG-HCG sequences treatment. Ann. Biol. anim. Bioch. Biophys., 15, 471-480.

Palmer E., Thimonier J., Lemon M., 1974. Early pregnancy diagnosis in the mare by estimation of the level of progesterone in the peripheral blood. Livestock Production Science, 1, I97-206.

SснӧNмUTн G., 1956. Vergleichende Untersuchungen über das Befrüchtungsvermögen der Warmbluthengste des Gestütes Neustadt/Dosse. Tierzucht, 10, 90-92.

Szumowski P., I964. Contrôle des facultés reproductrices de l'étalon. C. R. Acad. Agric., mai $1964,697-713$.

U.N.I.C., 1971, 1972, 1973, 1975. Abrégé des courses en France. Ed. Union Nationale Interprofessionnelle du Cheval. Vol. VII, 2 II-2I6, vol. VIII, 23I-237, vol. IX, 108-II2, vol. X, $164-170$, vol. XI, $236-243$. 